\title{
39. THE GALACTIC STRUCTURE AND THE APPEARANCE OF THE MILKY WAY
}

\author{
E. D. PAVLOVSKAYA and A. S. SHAROV \\ Moscow University, Moscow, U.S.S.R.
}

The appearance of the Milky Way for an observer situated within our Galaxy is determined by the spatial distribution of stars and absorbing interstellar matter. Hence it may be hoped that the study of the surface brightness of the Milky Way permits to derive the spiral structure of our Galaxy.

Some years ago Elsässer and Haug (1960) suggested a model of the galactic spiral structure based on an analysis of the distribution of the Milky Way surface brightness along the galactic equator. They have identified the bright parts of the Milky Way with the directions where the lines of sight run along the spiral arms. However Behr (1965) pointed out that, in the presence of interstellar absorption within the arms, the bright Milky Way parts might correspond to the directions between the arms.

We consider this problem in detail using different models of the galactic structure.

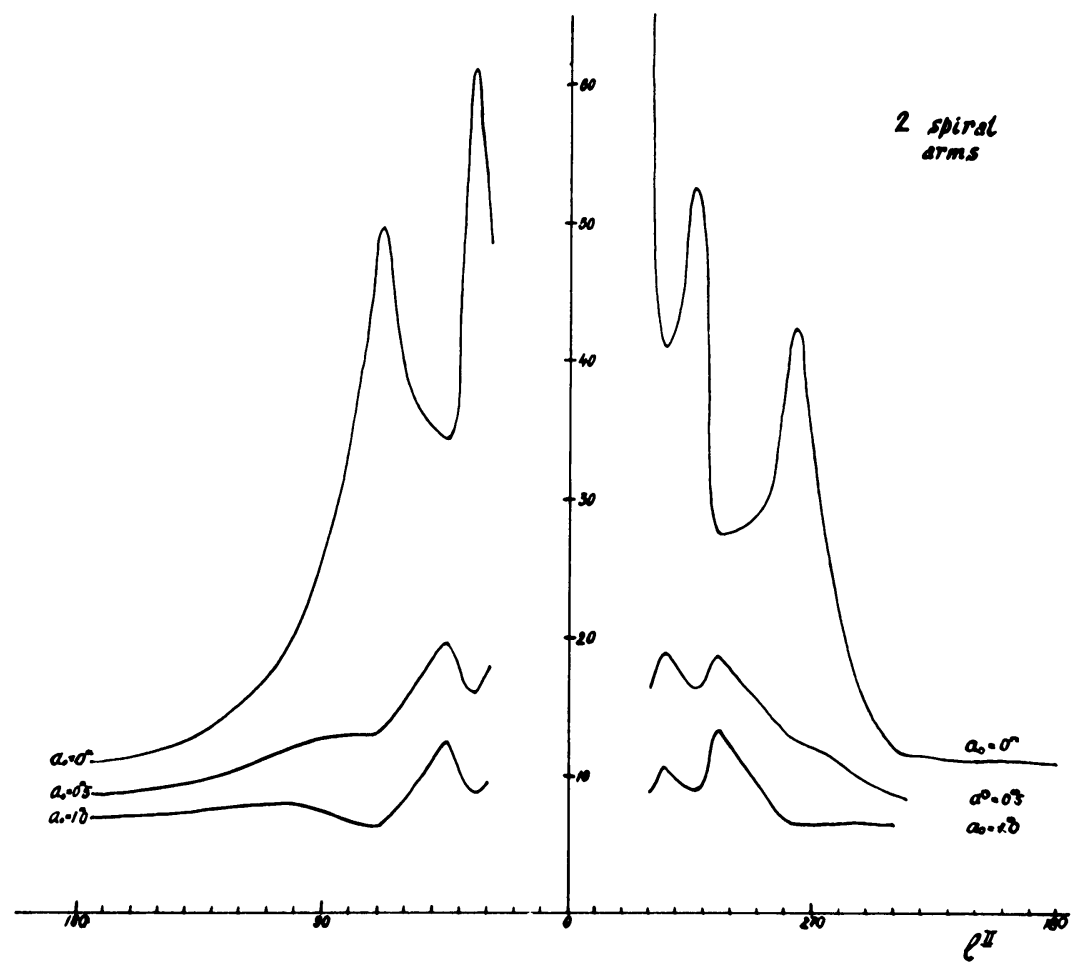

Fig. 1. The Milky Way brightness along the galactic equator in the 2 spiral arms model of Galaxy with different absorptions in the arms $\left(0^{\mathrm{m}}, 0^{\mathrm{m}} .5,1^{\mathrm{m}} .0 \mathrm{kpc}^{-1}\right)$. 
It is assumed that the model of our Galaxy consists of the disk with a radius of $15 \mathrm{kpc}$, and of several arms in the form of logarithmic spirals. The sun is situated in an arm at $10 \mathrm{kpc}$ distance from the centre of the system. We considered two geometrical models of the spiral arms - the model of Mills (1959) with 2 arms, and another one, with 14 arms derived by us (Pavlovskaya and Sharov, 1966). Our computational program permits to suppose the absorption either constant or variable across the spirals, and to change other parameters of our models. Results of calculations of the Milky Way brightness along the galactic equator for the two cases with 2 and 14 spiral arms $0.8 \mathrm{kpc}$ wide are represented in Figures 1 and 2. It is supposed that both the disk and the spirals have constant densities, taken to be equal to 1 and 5 , respectively.

The upper curves on both figures show the 'photometric profile' of the Milky Way if the interstellar absorption $a_{0}$ is zero. The two lower curves of each figure give the results of our calculations for constant absorption inside spiral arms: $a_{0}=0.5$ and $1 . \mathrm{m} 0 \mathrm{kpc}^{-1}$. In the absence of absorption the bright parts of the Milky Way correspond to the directions along the arms. However the presence of absorption leads to a change of the general appearance of the Milky Way. With acceptable values of the absorption the maxima of brightness shift in the directions between the spiral arms.

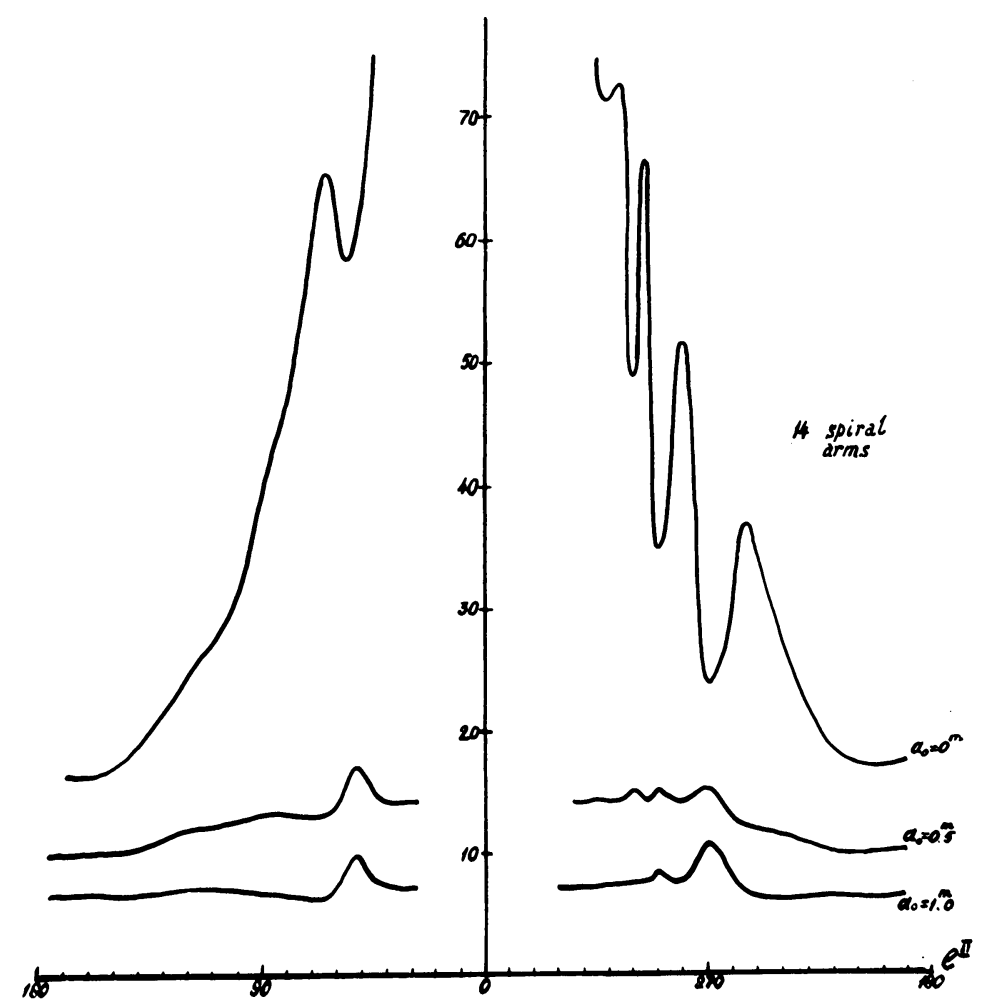

Fig. 2. The Milky Way brightness along the galactic equator in the 14 spiral arms model of Galaxy with different absorptions in the arms $\left(0^{\mathrm{m}}, 0^{\mathrm{m}} .5,1^{\mathrm{m}} .0 \mathrm{kpc}^{-1}\right)$. 
We considered a number of models with different values of absorption inside and between the arms as well as models with variable absorption across the arms. The analysis of all the results shows that even within the limits of our models the appearance of the Milky Way essentially depends on the value of absorption, and also on other parameters of the system. Of course, the real clouds of dark matter make the general appearance of the Milky Way more complicated. It seems unlikely that the study of surface brightness of the Milky Way might serve as a powerful tool for the investigation of the galactic structure. Hence it should not be supposed that the coincidence of the directions of the spiral arms, derived by other methods, is an essential argument in favour of any model of the galactic structure (Kardashev et al., 1964).

It should be noted that all the regular models do not explain the higher brightness of the southern Milky Way as compared with the northern one. The difference appears to be connected with different values of the mean absorption in the two mentioned areas of the Milky Way (Fernie, 1962; Sharov, 1963).

The complete work will be published in the Soviet Astronomical Journal.

\section{References}

Behr, A., 1965, Z. Astrophys. 62, 157.

Elsässer, H. and Haug, U.: 1960, Z. Astrophys. 50, 121.

Fernie, J. D.: 1962, Astron. J. 67, 224.

Kardashev, N. S., Lozinskaya, T. A., and Sleptsova, N. F.: 1964, Astron. Zh. 41, 601.

Mills, B. Y.: 1959, Publ. Astron. Soc. Pacific 71, 267.

Pavlovskaya, E. D. and Sharov, A. S.: 1966, Astron. Zh. 43, 40.

Sharov, A. S.: 1963, Astron. Zh. 40, 900. 